\title{
EFECTO DE LA FORMACIÓN DOCENTE INICIAL EN LAS CREENCIAS EPISTEMOLÓGICAS
}

\author{
Francisco Leal Soto \\ Universidad de Tarapacá, Chile
}

\section{INTRODUCCIÓN}

El Gobierno de Chile se encuentra comprometido desde 1990 en un proceso de reforma educativa de gran envergadura, con el objetivo de mejorar los niveles de calidad y equidad de los resultados pedagógicos del sistema educacional (García-Huidobro y Cox, 1999); esta reforma se inscribe en el contexto de los esfuerzos internacionales por promover una educación adecuada a las exigencias del actual y vertiginoso momento tecnológico, cultural y social que se experimenta en todo el mundo. (Delors, 1996). La importancia de los profesores en cualquier intento de reorganización educacional, desarrollo curricular o mejoramiento de la efectividad de la educación ha sido ampliamente reconocida (Calderhead, 1996), y no pasa inadvertida a los responsables de implementar esta reforma en Chile, quienes enfatizan que ésta "empieza a existir cuando sus lineamientos y orientaciones son reformuladas y convertidas, en las escuelas y liceos, mediante la energía y creatividad de los docentes, en propósitos compartidos y en prácticas pedagógicas renovadas" (García-Huidobro y Cox, 1999, p. 14). Los profesores actúan como intérpretes de ideas y portadores de valores, sujetos a sus experiencias anteriores, trabajando en instituciones que involucran complejas interacciones de ideas y acciones, por lo que son un factor clave en cualquier modificación curricular (Calderhead, 1996). Los desafíos que enfrentan los profesores en este proceso son, por una parte, involucrarse activamente en la reforma como un proceso del que son actores privilegiados; y por otra, modificar sus prácticas y sus formas de entender el aprendizaje y la enseñanza para hacerlos coherentes con el enfoque curricular y metodológico de la reforma (Núñez, 1999). En este caso específico, la reforma educativa en Chile intenta hacerse cargo del avance en la psicología cognitiva que ha puesto de relieve el papel de los seres humanos en la construcción de su propia realidad, permitiendo el desarrollo de teorías y técnicas de investigación que, al ser aplicadas a la investigación de los procesos de enseñanza, facilitan el surgimiento de modelos estructurales del conocimiento al destacar la interacción entre las cogniciones y las conductas de profesores y alumnos para explicar los procesos educativos (Calderhead, 1996; De Corte, 2003). Ninguno de estos desafíos parece logrado en Chile por los profesores en ejercicio, lo que ha sido adjudicado a una participación poco entusiasta y a la falta de interés de los profesores por las decisiones y prácticas pedagógicas (Núñez, 1999). Sin embargo, Lortie (1975) ha señalado que una de las razones por las cuales los programas de reforma curricular implementados en las últimas décadas no han sido exitosos podría ser la distancia entre las cogniciones de los profesores acerca del conocimiento, el aprendizaje y la enseñanza desarrolladas a lo largo de años de práctica al amparo de los modelos pedagógicos tradicionales, y los modelos cognitivos que fundamentan los componentes pedagógicos de la reforma. Los profesores tenderían a identificarse con los modelos y prácticas tradicionales, lo que generaría un efecto de continuidad más que de cambios.

De aquí la relevancia de influir en la formación inicial de los docentes. Al iniciarse el proceso de reforma, las instituciones chilenas de formación inicial de docentes presentaban currículos integrados por 
contenidos heterogéneos y fragmentados, con exceso de clases lectivas, escasas oportunidades de aprendizaje autónomo y escaso contacto con la realidad escolar, además de estrategias instruccionales contrarias a las que se les solicita aplicar como futuros profesores (Avalos, 1999). Por ello, uno de los programas de la reforma fue promover procesos de cambio en la formación inicial ofrecida por las instituciones formadoras, con la intención de modificar las posibilidades de actuación de los futuros profesores, favoreciendo el desarrollo de disposiciones, habilidades y conocimientos compatibles con las prácticas que la reforma exige. Como resultado, la mayor parte de las instituciones formadoras de profesores introdujo cambios en su currículo a partir de 1997, asumiendo una visión cognoscitivista del aprendizaje, distanciándose de la visión conductista vigente al inicio de la reforma; favoreciendo la enseñanza reflexiva, el aprender haciendo y la investigación para la acción; introduciendo oportunidades para tomar decisiones y desarrollar autonomía profesional; facilitando formas de aprendizaje colaborativo; y proponiendo organizaciones curriculares más integradas, interdisciplinarias y centradas en problemas más que en disciplinas (Avalos, 1999). A más de cinco años de su diseño y puesta en marcha, estas modificaciones sustantivas al proceso de formación han cumplido ya un ciclo completo, por lo que cabría esperar que estuvieran suficientemente consolidadas como para ejercer una influencia relevante sobre las creencias de los profesores en formación.

Las creencias son construcciones mentales a las cuales se les asigna suficiente validez, verdad o credibilidad como para guiar el pensamiento y la conducta (Harvey, 1986; Kitchener, 2002; Sigel, 1985). Pajares (1992) destaca que las creencias juegan un rol adaptativo, al facilitar a las personas su definición del mundo y de sí mismos. En el caso de los profesores, se ha señalado que sus predisposiciones personales constituyen el factor más relevante de su "ser profesor", y que sus experiencias como estudiantes les han conducido a desarrollar creencias que luego, cuando ellos mismos actúan como profesores, trasladan a la sala de clases (Lortie, 1975; Rokeach, 1968). Por su parte, Nespor (1987) señala que cuando un profesor encuentra un dominio confuso y las estrategias cognitivas y de procesamiento de información habituales no le dan buenos resultados, enfrenta la incertidumbre al no poder reconocer la información relevante y la conducta apropiada. Entonces, al no poder hacer uso de una estructura adecuada de conocimiento, el profesor recurre a las creencias, con sus limitaciones, problemas e inconsistencias. Este modo de funcionar es particularmente inadecuado en una profesión caracterizada por la cantidad y multiplicidad de contactos interpersonales que obligan a funcionar más por impulsos o intuición que por reflexión (Eraut, 1985; Jackson, 1968; Lortie, 1975). Destacando la función cognitiva que cumplen las creencias, Nespor (1987) las describió como ideas que definen las tareas, y prescribió una perspectiva del procesamiento cognitivo necesario para entender esta función: él plantea que las personas usan el pensamiento estratégico del tercer nivel (control, metacognición) para determinar las herramientas cognitivas a fin de enfrentar la tarea, y que es allí donde las creencias juegan un rol importante, al determinar la naturaleza de la tarea o definir el problema. Muy cercano a este planteamiento se encuentra el de Kitchener (1983), quien propuso un modelo de tres niveles para explicar el modo en que las personas se aproximan a problemas complejos: los dos primeros niveles corresponden respectivamente a la cognición y la metacognición, es decir, a los procesos de conocimiento y a los procesos de control de los mismos; al resolver problemas complejos, sin embargo, se pone en juego un tercer nivel, a través del cual se monitorea la naturaleza epistemológica del problema y el valor de verdad de las soluciones alternativas. En este nivel, se pone en juego el conocimiento acerca del conocimiento en un sentido propiamente epistémico, e incluye el conocimiento acerca de los límites del conocer, de la certeza del conocimiento, de los criterios del 
conocimiento válido y de las estrategias para identificar las formas requeridas para solucionar un problema y escoger las más adecuadas; es en este nivel donde se define la naturaleza de la tarea. Pajares (1992) concluye que las creencias, en general, son instrumentales para definir las tareas y seleccionar las herramientas cognitivas con las cuales interpretar, planear y tomar decisiones, por lo que son relevantes en la definición del comportamiento y en la organización del conocimiento y la información; y que las creencias epistemológicas, en particular, juegan un rol crucial en la interpretación del conocimiento y el monitoreo cognitivo.

Según Rockeach (1968), un sistema de creencias contiene representadas todas las creencias de una persona acerca de la realidad física y social, organizadas de una forma psicológica, pero no necesariamente lógica. Asume tres supuestos para esta organización: que las creencias difieren en intensidad y poder; que varían en una dimensión de centralidad-marginalidad, entendiendo la centralidad en términos de interconexiones con otras creencias; y que a mayor centralidad de la creencia, mayor resistencia cognitiva al cambio. Sugiere que determinar y comprender las conexiones funcionales entre creencias de distintos niveles puede ayudar a establecer la centralidad de algunas creencias, y facilitar la comprensión de las inconsistencias desde esta perspectiva. Las creencias epistemológicas son un conjunto particular, y muy relevante, de creencias. El concepto de creencias epistemológicas surge de la propuesta de Perry (1970) con respecto a la evolución del pensamiento de los estudiantes universitarios de pregrado (college) desde una perspectiva de creencias en verdades absolutas hasta aquellas que implican la consideración de diversas perspectivas que es necesario juzgar de acuerdo a criterios previamente establecidos. A partir de ese trabajo, se ha desarrollado un importante conjunto de investigaciones sobre las creencias acerca del conocimiento y el proceso de conocer, bajo diferentes denominaciones: creencias o teorías epistemológicas, modos de conocer, cognición epistémica, epistemología personal, entre muchas otras (Hofer, 2002). Hofer (2001) sostiene que la investigación en el área de la epistemología personal se ha centrado en el pensamiento y en las creencias de los estudiantes acerca del conocer y del conocimiento, e incluye creencias acerca de la definición del conocimiento, de cómo se construye, cómo se evalúa, dónde se ubica, y cómo ocurre, es decir, acerca de la naturaleza del conocimiento y de la naturaleza del conocer. El constructo de creencias epistemológicas y la investigación relacionada, han incluido también a las creencias acerca de la naturaleza del aprendizaje y la instrucción y acerca de la inteligencia, como lo comprueban Hofer y Pintrich (1997) en su revisión del tema. Estos autores señalan que sólo las creencias directamente atingentes al conocimiento y a la naturaleza del conocer corresponderían estrictamente al terreno epistemológico, y sugieren restringir el uso del concepto sólo a estos dos tipos de creencias. De acuerdo a ellos, la exclusión de creencias relativas a aspectos relacionados pero distintos del conocimiento, daría mayor claridad al concepto de creencias epistemológicas. Otros autores no están de acuerdo con este uso restringido del concepto de creencias epistemológicas; Moore (2002), por ejemplo, señala que, dado que el principal interés de la investigación en el tema es comprender el aprendizaje, este uso restringido del concepto deja fuera creencias relevantes. Sostiene, por lo tanto, que es necesario incluir en la investigación los aspectos relativos al aprendizaje. Refiriéndose específicamente al tratamiento del tema entre los profesores, Pajares (1992) constata que se ha usado el término creencias de los profesores para hacer alusión a sus creencias epistemológicas en el sentido amplio, en concordancia con lo planteado por Moore (2002). 
Aunque la investigación en este campo ha sido abundante y diversa tanto conceptual como metodológicamente (véase lo señalado por Hofer, 2002), y se han propuesto varios modelos para comprender las creencias epistemológicas y su desarrollo (Baxter-Magolda, 2002; Clinchy, 2002; Hofer y Pintrich, 1997; King y Kitchener, 1994, 2002; Kuhn y Weinstock, 2002; Moore, 2002; Schommer-Aikins, 2002), la mayor parte de los autores, por lo menos, comparten un elemento básico: ellos asumen que las creencias epistemológicas pueden caracterizarse en términos de la sofisticación para entender la realidad, entre los extremos marcados por una comprensión objetiva directa y una comprensión subjetiva mediada por marcos de referencia que pueden ser evaluados, y que corresponderían a bs extremos de menor y mayor desarrollo evolutivo respectivamente (Moshman, 1998). Desde una perspectiva distinta, Schommer (1990) criticó la proposición de que las creencias epistemológicas fueran unidimensionales y secuenciadas, proponiendo que son más bien un sistema de cinco o más dimensiones relativamente independientes entre sí, y que no siguen una estricta secuencia en su desarrollo: estructura, certeza y origen del conocimiento, y control y rapidez del aprendizaje. Al interior de cada una de estas dimensiones, sin embargo, se reconoce el continuo que va desde la posición en que el conocimiento se conceptualiza como simple y certero y el aprendizaje como inmediato e incontrolable por el sujeto, hasta aquélla en que el conocimiento se concibe como tentativo y complejo, y el aprendizaje gradual y controlado por el sujeto (Schommer-Aikins, 2002). Así, una misma persona podría presentar creencias cercanas a una posición en algunas dimensiones y cercanas a la otra posición, en otras.

En un trabajo reciente sobre la relación entre creencias y prácticas educativas en profesores, Schraw y Olafson (2003) utilizaron el concepto de cosmovisión epistemológica. Para ellos, cosmovisión epistemológica se refiere a un conjunto de creencias que definen la actitud de una persona hacia la naturaleza y la adquisición del conocimiento, concepto bastante similar al de las orientaciones para la enseñanza, de Porter y Freeman (1986). Cada persona tiene un conjunto de creencias epistemológicas, pero éstas a su vez son parte de una cosmovisión epistemológica, que puede incluir otras creencias relacionadas, por ejemplo, acerca de cómo se adquieren, desarrollan o cambian las propias creencias. La cosmovisión epistemológica sería la suma de las creencias y supuestos de una persona que influyen en el modo en que ella se acerca al conocimiento; es un concepto amplio que sugiere una perspectiva intelectual que actúa como un lente a través del cual se mira el mundo, y que trasciende las creencias específicas sobre el conocimiento. Los profesores que sostienen la cosmovisión realista asumirían que existe un cuerpo de conocimientos objetivos, al que se accede a través de los expertos por transmisión y reconstrucción. Este conocimiento sería compartido por los expertos y es relativamente estable. Los profesores que tienen esta perspectiva serían enseñantes activos, mientras los estudiantes tendrían un rol receptivo y pasivo, para lo cual la práctica deliberada sería una estrategia privilegiada. Los profesores tenderían a evaluar con referencia a normas, ya que enfatizarían la comparación entre estudiantes en términos de cantidad de objetivos o contenidos curriculares alcanzados, y serían proclives a utilizar evaluaciones preparadas por expertos externos. Además, asignarían escasa importancia al rol de los pares en el aprendizaje. Desde la cosmovisión relativista, en cambio, se asume que cada aprendiz construye su propio y único conocimiento, por lo que éste sería subjetivo y altamente modificable. Los profesores que sostienen esta perspectiva tenderían a negar la superioridad de cualquier conocimiento, incluso el propio, por lo que enfatizarían su rol en la creación de ambientes en los cuales los estudiantes desarrollen su propio pensamiento. Su objetivo principal sería facilitar la autorregulación del estudiante para capacitarlo para el autoaprendizaje. Su evaluación haría referencia a criterios desarrollados para cada estudiante, y darían importancia a la 
autoevaluación. Los pares no serían particularmente relevantes. Desde la cosmovisión contextualista, finalmente, se supone que los aprendices construyen conocimiento colectivamente en contextos de colaboración. Los profesores en esta perspectiva se percibirían como facilitadores, y se preocuparían tanto del tipo de conocimiento construido como del proceso de construcción y de la pertinencia del conocimiento en los contextos propios de los alumnos. El conocimiento sería visto como modificable de acuerdo a las necesidades de cada situación. Utilizarían evaluación cooperativa en actividades de aprendizaje, y promoverían la interacción y el apoyo entre pares. Aunque se plantean como discretas entre sí, estas tres cosmovisiones corresponden a aquellas posiciones más frecuentemente identificadas por los investigadores en el continuo de la sofisticación de los supuestos con que se asume el conocimiento.

Desde el punto de vista curricular, los principios de la reforma educacional chilena se acercan a la perspectiva subjetivista y se asumen como orientadoras las teorías del aprendizaje que tienen como centro el concepto de aprendizaje significativo y dan gran importancia a la experiencia previa y al sentido que para los alumnos tiene cada nuevo aprendizaje (García-Huidobro y Cox, 1999). Según Cox (1999), con la reforma se asume una visión generativa del conocimiento, se enfatiza en su contextualidad y significatividad del conocimiento, y los saberes se reconocen como empresas humanas, históricas, lo que lleva a plantear como contenido no sólo los saberes en sí mismos, sino también los procesos de su construcción, incluyendo las diferentes visiones, las disputas, y los avatares sociales y prácticos que los constituyeron. En términos metodológicos, con el marco curricular se proponen procedimientos didácticos centrados en la actividad de los alumnos, tanto individual como colaborativa, a través de los cuales se busca favorecer el desarrollo de capacidades y habilidades superiores como análisis, interpretación y síntesis de información de diversas fuentes, abstracción y elaboración de conocimientos, capacidad para pensar en sistemas, comprensión de procesos y fenómenos, resolución de problemas, capacidad de experimentar y aprender a aprender, comunicación efectiva, trabajo en equipo, manejo de la incertidumbre y adaptación a los cambios. Esta propuesta exige de los profesores habilidades, disposiciones y prácticas que son muy distintas de las exigidas por los modelos curriculares en vigencia al inicio de la reforma, basados en los planteamientos de Bloom (1971) que ponen el énfasis en los objetivos conductuales, en la tecnología educativa orientada hacia el logro y encaminados más hacia las relaciones de control que hacia los saberes y valores a trasmitir. Estos requerimientos descritos por Cox están más cercanos a las creencias señaladas por Schraw y Olafson (2003) dentro de las cosmovisiones relativista y contextualista, diferenciándose claramente de la realista. A su vez, estas cosmovisiones se corresponden con el lado más sofisticado, más subjetivista, de la dimensión básica en que coinciden la mayor parte de los principales investigadores de las creencias epistemológicas (Baxter-Magolda, 2002; Clinchy, 2002; Hofer y Pintrich, 1997; King y Kitchener, 1994, 2002; Kuhn y Weinstock, 2002; Moore, 2002; Schommer-Aikins, 2002).

Considerando que la formación inicial de los profesores en Chile se ha modificado explícitamente para hacerse coherente con esta perspectiva (Avalos, 1999), cabría esperar que los profesores en formación desarrollaran creencias coherentes con la perspectiva subjetivista durante su proceso de entrenamiento. Al respecto, Collier (1999) señala que los profesores en formación comienzan a construir realidad acerca de la enseñanza con su experiencias como alumnos y aprenden a acceder y hacer explícitas sus creencias a través de preguntas y respuestas relevantes para asimilar tanto su rol como profesores como las características de la enseñanza eficaz. Las reflexiones durante este período les ayudarían a descubrir y sintetizar ideas desde una perspectiva personal (Canning, 1991). Algunos estudios 
sugieren que se puede ayudar a los estudiantes a reflexionar y valorar la reflexión sobre la enseñanza y el aprendizaje (Pultorak, 1993, 1996; Rudney y Guillaume, 1990; Wildman y Niles, 1987). Schraw y Olafson (2003) coinciden en señalar que la formación inicial de los profesores es uno de los factores que contribuyen al desarrollo de su cosmovisión epistemológica. Por otra parte, Freeman (1991) sugiere que un elemento importante de la formación docente es el proceso de hacer explícitos los sistemas implícitos de creencias y desarrollar un lenguaje para conversar y pensar sobre su propia práctica, cuestionando sus creencias contradictorias y tomando mayor control sobre su desarrollo profesional. Pajares (1992), en cambio, señala que las creencias acerca de la enseñanza están bien establecidas cuando los alumnos ingresan al pregrado universitario (college), y argumenta que ellas son difíciles de modificar en los procesos de formación. Considerando las evidencias proporcionadas por varios autores, Brownlee, Purdie y BoultonLewis (2001) han destacado que puede ser muy lento y difícil intervenir para cambiar las creencias. Rockeach (1968), por su parte, ha propuesto que mientras más conectada está una creencia dentro de la estructura actitudinal, es más central y difícil de cambiar; además señala que la experiencia como estudiante sirve para desarrollar creencias que luego son trasladadas a la sala de clases cuando ejercen como profesores. En el caso específico de la formación de profesores, Pajares (1992) argumenta que esta dificultad para el cambio de las creencias podría deberse a la familiaridad de los estudiantes con los procesos de enseñanza durante su experiencia como alumnos en la escuela. En la mayoría de los estudios universitarios, los estudiantes son ajenos al campo específico de estudio cuando recién ingresan, por lo que carecen de preconceptos o creencias previas acerca de las disciplinas (Posner, Strike, Hewson y Gertzog, 1982). Los estudiantes de pedagogía, en cambio, no son nuevos en el campo de estudio: las prácticas de formación difieren muy poco de su experiencia de escolarización previa, por lo que no deben redefinir ni recrear; hay continuidad con la experiencia anterior, por lo que sus expectativas, conocimientos y creencias previas les resultan pertinentes. Adquirir nuevos conocimientos o cambiar creencias, para ellos, puede resultar difícil, conflictivo o amenazante (Schutz, 1970). Ginsburg y Newman (1985) plantean que esta familiaridad con el contexto juega un rol importante en el proceso político de reproducción social; la mayor parte de los estudiantes de pedagogía se identifican con las prácticas educativas, lo que los lleva a la mantención de dichas prácticas y hacia la continuidad más que al cambio (Lortie, 1975).

Lonka, Joram y Bryson (1996) presentaron los resultados de dos estudios realizados para determinar si el entrenamiento produce cambios en las creencias sobre el aprendizaje y el conocimiento, a través de una tarea de definición y una de resolución de problemas. El primero, crosseccional, mostró que mientras más entrenamiento en educación tenían los sujetos, mayor era su tendencia a producir definiciones constructivistas; sin embargo, en la tarea de solución de problemas, sólo los expertos fueron consistentes en producir soluciones constructivistas. Los resultados del estudio longitudinal mostraron que hay un cambio significativo en las definiciones emitidas por los estudiantes antes y después de un curso de Psicología Educacional, siendo más constructivistas las del postest; sin embargo, al solucionar un problema concreto no se evidenciaron cambios entre pre y post test, es decir las respuestas no fueron constructivistas, inclusive en los profesores. Una de las implicaciones discutidas fue que los expertos y los no expertos tienen ideas distintas acerca del aprendizaje; y que ésta puede ser una de las razones de las dificultades para implementar reformas pensadas solamente desde el marco de referencia de los expertos. Por su parte, Hill (2000) desarrolló una experiencia educativa con estudiantes de pedagogía de un curso de Psicología Educacional, basada en la idea de comunidad de aprendizaje. Con un diseño cuasi experimental, con medidas cuantitativas y cualitativas, encontró un claro efecto del programa luego de 16 semanas de 
duración, produciéndose una mejoría de medio estadio en el esquema de Perry en los alumnos del grupo experimental, y ningún cambio en el grupo control. Brownlee et al. (2001) presentaron un trabajo realizado con estudiantes de pregrado en pedagogía, a quienes se sometió a un proceso de lecturas, discusión y reflexión sobre las creencias epistemológicas y sus propias creencias, como parte de un curso anual; y a un grupo control se le sometió al mismo curso, pero sin el énfasis en las creencias epistemológicas. Se evaluó a los sujetos antes y después del curso. Los resultados arrojaron diferencias significativas entre los grupos, con un mayor avance del grupo experimental en el transcurso del año; los autores interpretaron este resultado como producto de un desequilibrio cognitivo que favoreció la reflexión epistémica. Considerando este conjunto de evidencias, no resulta claro que la modificación del proceso de formación docente inicial resulte necesariamente en profesores cuyas creencias sean concordantes con el enfoque subjetivista requerido por la reforma. El objetivo de este estudio fue determinar si efectivamente el proceso de formación inicial docente en una universidad chilena, explícitamente orientado por el enfoque subjetivista, produce un desplazamiento de las creencias de sus alumnos hacia el polo subjetivista en el continuo epistemológico. La hipótesis fue que los alumnos de niveles más avanzados en su formación pedagógica mostrarían mayor presencia de creencias caracterizables como subjetivistas que los alumnos de niveles menos avanzados y, por tanto, menos expuestos a una formación explícitamente orientada por ese tipo de creencias.

\section{MÉTODO}

\section{Muestra}

Éste es un estudio cross-seccional en que la muestra estuvo conformada por 123 alumnos de primer y tercer nivel de las carreras de Pedagogía en Educación General Básica, Pedagogía en Matemáticas y Computación y Pedagogía en Historia y Geografía, de una universidad chilena de carácter público, ubicada en una ciudad de 200.000 habitantes. La muestra fue tomada por participación voluntaria; la composición de la muestra por carrera, sexo y nivel, se muestra en la Tabla № 1. El promedio de edad para el primer nivel fue de 21.63 años, con una mediana de 21, desviación estándar de 3.98 y valores extremos de 17 y 40 años. Para el tercer nivel, el promedio de edad fue de 22.35 años, con una mediana de 22, desviación estándar de 3.52 y valores extremos de 18 y 33 años.

Tabla № 1

Composición de la muestra por nivel, carrera y sexo

\begin{tabular}{|c|c|c|c|c|c|}
\hline \multirow[b]{2}{*}{ Nivel } & \multirow[b]{2}{*}{ Sexo } & \multicolumn{3}{|c|}{ Carrera } & \multirow[t]{2}{*}{ Total } \\
\hline & & $\begin{array}{c}\text { Educación } \\
\text { General Básica }\end{array}$ & $\begin{array}{l}\text { Matemáticas y } \\
\text { Computación }\end{array}$ & $\begin{array}{l}\text { Historia y } \\
\text { Geografía }\end{array}$ & \\
\hline \multirow[t]{3}{*}{ Primero } & Hombre & 4 & 23 & 15 & 42 \\
\hline & Mujer & 24 & 25 & 12 & 61 \\
\hline & Total & 28 & 48 & 27 & 103 \\
\hline \multirow[t]{3}{*}{ Tercero } & Hombre & 2 & 14 & 23 & 39 \\
\hline & Mujer & 15 & 9 & 27 & 51 \\
\hline & Total & 17 & 23 & 50 & 90 \\
\hline \multicolumn{2}{|c|}{ Gran total } & 45 & 71 & 77 & 193 \\
\hline
\end{tabular}

\section{Instrumento}

Aunque existen instrumentos para evaluar creencias epistemológicas, ellos no están validados en el contexto cultural chileno. La información empírica disponible advierte sobre los riesgos de utilizar 
instrumentos de evaluación de creencias en contextos culturales diferentes a los originales, ya que se ha señalado y encontrado que la cultura influye en las formación de las creencias (Alexander y Dochy, 1995; Hofer y Pintrich, 1997) y que los instrumentos podrían ser inadecuados en contextos culturales diferentes a aquél en que fueron desarrollados (Arredondo y Rucinski, 1996; Chan y Elliot, 2002; Zhang, 1999). Por esta razón, se optó por desarrollar un instrumento específico para esta investigación, considerando como objeto de evaluación las creencias epistemológicas en el sentido amplio defendido por Moore (2002). Para esto se tomaron como referencia básica las creencias descritas en las tres cosmovisiones epistemológicas propuestas por Schraw y Olafson (2003) y en el Epistemological Beliefs Inventory (Schraw, Bendixen y Dunkle, 2002), reelaboración de la propuesta de Schommer (1990). A partir de estos modelos, se elaboró un conjunto de afirmaciones que reflejaban estas creencias, el que fue presentado a cuatro jueces (un Doctor y tres Licenciados en Psicología), quienes recibieron una breve descripción de los modelos y los escritos in extenso en que originalmente éstos se presentaron. Cada afirmación fue presentada como perteneciente a una cosmovisión y a un tipo de creencia. La tarea que se pidió a los jueces fue manifestar su acuerdo o desacuerdo con la pertenencia de cada afirmación a la cosmovisión a la que fue inicialmente adscrita, así como formular observaciones respecto de la validez aparente y los aspectos formales. La retroalimentación de estos jueces permitió eliminar o modificar las afirmaciones que fueron estimadas poco claras o incongruentes con los modelos, disponiéndose finalmente de treinta y un afirmaciones no objetadas por ninguno de los jueces. Este conjunto fue sometido a una nueva evaluación, esta vez por cuatro profesores en ejercicio, a quienes se les solicitó su opinión respecto de la claridad y la validez aparente de las afirmaciones. Como resultado, dos afirmaciones fueron eliminadas ya que implicaban una opinión directa sobre los profesores.

Debido a que en todos los estudios se ha encontrado pocos sujetos en los niveles más altos en el continuo de las creencias epistemológicas (aquellos que implican el reconocimiento sistemático de la evaluación contextual) en poblaciones no especializadas (Hofer y Pintrich, 1997), se clasificaron todas las afirmaciones correspondientes a las cosmovisiones relativista y contextualista como subjetivistas, y las afirmaciones correspondientes a la cosmovisión realista, como objetivistas. De esta manera, se dispuso de veintinueve afirmaciones, de las cuales dieciocho correspondieron a la perspectiva subjetivista y once a la objetivista. Estas afirmaciones fueron ordenadas aleatoriamente y presentadas como opiniones de profesores recogidas en situaciones escolares. La tarea solicitada a los sujetos fue manifestar su acuerdo o desacuerdo con cada afirmación, en una escala tipo Likert de cinco puntos, en que uno correspondía a total desacuerdo y cinco a total acuerdo. Por razones de claridad teórica y metodológica, y dado que el interés específico de este trabajo era evaluar si los profesores en formación modifican sus creencias en el sentido de la perspectiva subjetivista, se optó por crear una escala homogénea de subjetivismo; por esta razón, aunque siempre se presentó todas las afirmaciones a los sujetos, el análisis subsecuente se realizó sólo sobre las dieciocho afirmaciones correspondientes a la perspectiva subjetivista. De esta manera se puede obtener una puntuación global de subjetivismo sumando las puntuaciones drectas de todos ellos. Este instrumento fue aplicado a una muestra piloto de 65 profesores en ejercicio, que accedieron voluntariamente a colaborar cuando se les contactó en sus escuelas y en programas de capacitación en los que participaban. Se obtuvo la correlación biserial puntual de cada afirmación con la puntuación total, y se seleccionaron aquéllas que tuvieron una correlación ítem-total igual o superior a 0.20. Diez cumplieron con ese criterio; y, aunque se esperaba que una mayor cantidad de ellos pudieran ser seleccionados, un análisis cualitativo tanto de las afirmaciones descartadas como de las seleccionadas, reveló que las afirmaciones 
seleccionadas lograron una mayor proximidad con la única afirmación directamente relacionada con el conocimiento, incluida en el conjunto claramente relativista. Se dispuso así de una escala homogénea, formada por diez ítemes, cuya confiabilidad fue de 0.80 (Coeficiente Alfa de Cronbach), y cuya puntuación fluctuaba entre 10 y 50 puntos (Tabla №2).

Tabla № 2

Afirmaciones incluidas en la escala definitiva

La única alternativa para establecer lo correcto en estos asuntos es ponernos de acuerdo en los criterios que vamos a usar.

El verdadero aprendizaje consiste en que los alumnos desarrollen sus propios criterios personales. Creo que los alumnos deben aprender a juzgar lo que dicen los textos de acuerdo a su propia realidad.

La verdad depende de cómo se miren las cosas. Cada cual tiene su propia perspectiva.

El aprendizaje se logra efectivamente cuando los alumnos tienen la posibilidad de contrastar sus propias ideas con las ideas científicas y establecer relaciones entre ambas.

Yo prefiero que los alumnos investiguen y conversen sobre los términos relevantes, así establecen relaciones a partir de distintos puntos de vista aportados por ellos mismos.

Creo que es bueno permitirles que comenten entre ellos, así aprenden unos de otros.

Yo creo que hay que ir de a poco, y permitir que aprendan a su ritmo. Nadie aprende de una sola vez.

Prefiero que siempre haya varias perspectivas, para que los propios alumnos vayan evaluando las alternativas.

Me parece bien que no crean a pie juntillas lo que dicen los textos, y planteen visiones diferentes.

Adicionalmente, se pidió a los sujetos que proporcionaran información demográfica básica respecto

a sexo, edad, carrera y nivel de estudios.

\section{Procedimiento}

El instrumento fue presentado como una encuesta de opiniones sobre el aprendizaje, y aplicado en la segunda mitad del año académico, en conjunto con una batería de instrumentos correspondientes a otras investigaciones relacionadas. Las aplicaciones fueron realizadas al finalizar una hora de clases, con la autorización del profesor a cargo. La participación fue voluntaria, permitiéndose a los alumnos que no quisieron responder el instrumento hacer abandono de la sala antes de empezar la aplicación. Una vez recogidos los datos, se obtuvo la puntuación global para cada sujeto sumando las puntuaciones de los diez ítemes.

\section{RESULTADOS}

El promedio en la escala de subjetivismo para los alumnos de primer año fue de 41.85 puntos, en tanto que para los del tercer nivel fue de 43.52, con desviaciones estándar de 5.85 y 4.9 puntos respectivamente. Como se aprecia, ambos grupos presentaron altas puntuaciones en la escala, pero la diferencia a favor del tercer nivel fue significativa $(\mathrm{t}=-2.168, \mathrm{gl}=190.684$, sig=0.031, prueba de dos colas, varianzas iguales no asumidas). Sin embargo, debido a que la distribución de la muestra por carrera, edad y sexo resultó muy dispar entre los niveles, esta diferencia podría obedecer a estas variables más que al efecto del nivel. Para analizar esta posibilidad, se efectuó un análisis de varianza con las variables nivel, sexo y carrera como factores, y la edad como covariable. En este análisis, se perdieron diez casos que no registraron su edad, y por esto, los resultados difirieron ligeramente con los de la muestra total (Tabla № 3). 
Tabla № 3

Análisis de varianza de la puntuación en la escala de subjetivismo, por nivel, carrera y sexo, con la edad como covariable.

\begin{tabular}{|c|c|c|c|c|c|}
\hline Fuente de variación & $\begin{array}{c}\text { Suma de } \\
\text { cuadrados } \\
\text { (Tipo III) }\end{array}$ & gl & $\begin{array}{c}\text { Media } \\
\text { cuadrática }\end{array}$ & $\mathrm{F}$ & Sig. \\
\hline Modelo corregido & 990.549 & 12 & 82.546 & 3.406 & .000 \\
\hline Intercepción & 8458.596 & 1 & 8458.596 & 349.057 & .000 \\
\hline EDAD & $6.785 \mathrm{E}-02$ & 1 & $6.785 \mathrm{E}-02$ & .003 & .958 \\
\hline NIVEL & 206.395 & 1 & 206.395 & 8.517 & .004 \\
\hline CARRERA & 185.079 & 2 & 92.539 & 3.819 & .024 \\
\hline SEXO & 90.091 & 1 & 90.091 & 3.718 & .056 \\
\hline NIVEL * CARRERA & 284.761 & 2 & 142.381 & 5.876 & .003 \\
\hline NIVEL * SEXO & 79.529 & 1 & 79.529 & 3.282 & .072 \\
\hline CARRERA * SEXO & 2.240 & 2 & 1.120 & .046 & .955 \\
\hline NIVEL * CARRERA * SEXO & 50.183 & 2 & 25.092 & 1.035 & .357 \\
\hline Error & 4119.560 & 170 & 24.233 & & \\
\hline Total & 340217.000 & 183 & & & \\
\hline Total corregido & 5110.109 & 182 & & & \\
\hline
\end{tabular}

Estos resultados deben considerarse con precaución, ya que el distinto tamaño de los grupos y de sus varianzas pudieran producir distorsiones en la probabilidad asociada al estadístico (Ferrán, 1996). El efecto principal del nivel se mantuvo, independientemente de los efectos principales de carrera y sexo; este último, incluso, no alcanza significación por sí solo, aunque esto pudiera deberse al efecto de los tamaños de los grupos y a la desigualdad de las varianzas. De hecho, al analizar separadamente el efecto del sexo con la prueba $t$ de Student, éste resultó altamente significativo $(t=-2.310, g l=148.156, p=0.022$, prueba de dos colas, varianzas iguales no asumidas). Asimismo, se observa una interacción significativa entre nivel y carrera, y una tendencia en tal sentido, que no alcanza significación estadística, entre nivel y sexo. Finalmente y a modo de referencia, se realizó una comparación entre los dos grupos de alumnos y los 65 profesores que participaron en la aplicación piloto del instrumento, cuyo promedio fue de 40.42 con una desviación estándar de 5.59. Con los mismos resguardos que en el análisis anterior, debido a las diferencias de varianza y tamaño de los grupos, el análisis de varianza arrojó resultados signiticativos ( $F=6.2222$, $\mathrm{p}=0.002)$. En el análisis post-hoc con la técnica de Scheffé se encontró que el promedio de los profesores fue significativamente menor que el de los alumnos de tercer nivel, pero no presentaron diferencias significativas con el promedio de los alumnos de primer nivel.

\section{DISCUSIÓN}

En primera instancia, cabe destacar, que la alta puntuación obtenida por ambos grupos en la escala de subjetivismo, revela una presencia importante de creencias subjetivistas, correspondientes principalmente a la cosmovisión relativista descrita por Schraw y Olafson (2003) y al nivel caracterizado por Kuhn y Weinstock (2002) como multiplipicista. Estos últimos autores plantean que los valores de aceptación de la diversidad y la tolerancia individual imperantes en la sociedad promueven justamente el tipo de creencias epistemológicas que afirman la subjetividad individual como criterio esencial de validez, por lo que no resulta extraño el alto grado de acuerdo de ambos grupos con la perspectiva subjetivista evaluada por la escala. No obstante, la hipótesis que sostiene que las creencias educacionales de los profesores en formación se modifican como producto del proceso de formación inicial, en el sentido de aproximarse a una perspectiva subjetivista, resulta claramente soportada por los datos recogidos. El aumento en la puntuación de acuerdo con la perspectiva subjetivista entre el primer y el tercer nivel es significativo, y el efecto del 
nivel, aunque interactúa con otras variables, es un efecto independiente claramente reconocible. Este resultado es concordante con los estudios respecto al cambio en las creencias educacionales de los profesores durante su formación cuando hay un esfuerzo sistemático en tal sentido, como se desprende de los resultados reportados por Brownlee et al. (2001), Hill (2000), y Lonka et al. (1996), ya que la actual formación que reciben los estudiantes de pedagogía en Chile puede considerarse orientada en tal sentido: si bien tal vez no se ha panteado explícitamente el tema de las creencias educacionales, los supuestos básicos sobre los que descansa el currículo y el discurso explícito de la reforma apuntan en la dirección de la perspectiva subjetivista.

Sin embargo, es necesario considerar las limitaciones impuestas por el diseño cross-seccional, que no asegura la igualdad inicial de las cohortes. Frente a este elemento de duda, la comparación con el grupo de profesores aporta una evidencia adicional: los profesores de la muestra piloto fueron formados antes del proceso de reforma, con arreglo a los modelos tradicionales correspondientes a la perspectiva objetivista; por ello, el distanciamiento de los alumnos de tercer nivel respecto de los profesores puede ser interpretado como resultado de las modificaciones introducidas en el proceso de formación, mientras que la proximidad entre los profesores y los alumnos del primer nivel, obedecería al resultado de la consistencia entre la formación que recibieron los profesores y la experiencia de los mismos alumnos, en su proceso escolar previo a la formación pedagógica. Por otra parte, Veenman (1984) ha demostrado que los estudiantes de pedagogía pueden liberalizar sus formas de pensar durante su formación, pero que vuelven a una orientación conservadora una vez que ésta finalice, hecho que se relaciona hipotéticamente con la naturaleza contextual de las creencias; es posible pensar que la menor proximidad de las creencias educacionales de los profesores en ejercicio con los estudiantes del tercer nivel, sea resultado de esta "vuelta atrás" debido al cambio de contexto. En el mismo sentido, Schraw y Olafson (2003) han encontrado que la mayor experiencia como profesor se asocia con una mayor creencia en la simplicidad del conocimiento y en la autoridad -las que se asimilan a la perspectiva objetivista- y con menor acuerdo con la perspectiva contextualista.

Cualquiera sea la explicación asumida para este resultado obtenido en los profesores en ejercicio, las diferencias observadas en los estudiantes entre el primer y el tercer nivel, si se descarta el efecto de cohorte posible por el diseño, pero poco probable por la similitud de los contextos de un año a otro, pudieran ser atribuidas razonablemente al proceso formativo. Este cambio, sin embargo, no parece homogéneo para todas las carreras. Los estudiantes de Pedagogía en Historia y Geografía parecieran estar más cercanos a la perspectiva subjetivista que los de Educación General Básica y los de Matemáticas y Computación al iniciar la formación, y no modifican su perspectiva durante ese proceso. Esto es concordante con la apreciación de que los alumnos que escogen esa carrera, en particular, suelen mostrar desde el comienzo un mayor nivel de pensamiento crítico y actitud activa frente al aprendizaje, lo que se ve reflejado en mayor participación en clases y en la vida estudiantil en general, conductas que podrían también relacionarse con la forma en que este tipo de estudiantes se aproximan y conciben los respectivos dominios. Al respecto, Buehl y Alexander (2002), sintetizan los planteamientos de varios autores, al señalar que los dominios suelen ser teóricamente conceptualizados de manera diferente, y como más o menos estructurados por las diferentes disciplinas. Las disciplinas más estructuradas son vistas, con mayor frecuencia, enfrentando problemas o tareas con soluciones convencionales, derivadas de procedimientos o algoritmos, que las menos estructuradas; los problemas en disciplinas menos estructuradas son resueltos a través de métodos 
heurísticos que ayudan, pero no aseguran soluciones viables. Es posible pensar que, dadas estas diferentes percepciones acerca de dominios como Historia y Matemáticas, ellos resulten diferencialmente atractivos para jóvenes con creencias diferentes.

Resumiendo los resultados de este estudio, podría concluirse tentativamente que es posible reconocer un conjunto de creencias educacionales ligadas a una creencia epistemológica específica, caracterizables como perspectiva pedagógica subjetivista, y que la participación en un programa de formación inicial de profesores aumenta la aproximación a este tipo de perspectiva pedagógica, observándose pequeñas diferencias asociadas al tipo de disciplina. Estos resultados son consistentes con el objetivo de las modificaciones curriculares en que se han comprometido las universidades formadoras de profesores en el país, tendientes a dar una formación coherente con los principios cognitivos orientadores de la reforma educacional en curso, la que asume supuestos esencialmente subjetivistas. Sin embargo, las limitaciones del estudio obligan a plantear al menos dos consideraciones sobre el trabajo futuro necesario si se quiere establecer conocimiento más preciso y fundado al respecto. La primera, dice relación con el instrumento, y tiene ribetes teóricos y metodológicos. Por razones de simplicidad teórica y práctica, se optó por trabajar con un instrumento centrado en una perspectiva pedagógica única, la subjetivista, que corresponde aproximadamente con el modelo orientador de la reforma. Esta opción, sin embargo, impide establecer si es posible identificar otras perspectivas, y si es posible, cabría preguntarse ¿Cuál es la relación entre ellas? ¿Son excluyentes entre sí, al modo de lo planteado en el modelo de cosmovisión epistemológica de Schraw y Olafson (2003)?; o si, dado su nivel intermedio de especificidad y en concordancia con lo planteado por Hammer y Elby (2002), ¿Operan más bien como recursos disponibles en mayor o menor medida?; o bien, como señala Sinatra (2002), ¿Son creencias que evolucionan pero no desaparecen, sino que permanecen disponibles? Por esto, es indispensable trabajar en el desarrollo de un instrumento que permita evaluar simultáneamente un conjunto mayor y más diverso de creencias tanto educacionales como epistemológicas. La segunda consideración es de carácter estrictamente metodológico. El diseño cross-seccional tiene limitaciones intrínsecas, que impiden obtener conclusiones sólidas. La alternativa es la realización de estudios longitudinales, pero éstos son difíciles de llevar a la práctica. En primera instancia, sería conveniente intentar la realización de estudios longitudinales al respecto, pero, alternativamente, es necesario buscar estrategias para superar las limitaciones de los estudios crossseccionales, tales como el estudio de varios grupos simultáneamente, o estableciendo hipótesis más específicas que puedan ser contrastadas en subgrupos de sujetos (León y Montero, 1997).

\section{REFERENCIAS}

ALEXANDER, P. y DOCHY, F. (1995) Conceptions of knowledge and beliefs: A comparison across varying cultural and educational communities. American Educational Research Journal, 32, 413-442.

ARREDONDO, D. y RUCINSKI, T. (1996) Epistemological Beliefs of Chilean Educators and School Reform Efforts. Ponencia presentada en el Tercer Encuentro Nacional de Enfoques Cognitivos Actuales en Educación. Santiago, Chile: Noviembre 7-8.

AVALOS, B. (1999) Mejoramiento de la formación inicial docente. En J. García-Huidobro (Ed.) La reforma educacional chilena. Madrid: Editorial Popular, 195-214.

BAXTER MAGOLDA, M. (2002) Epistemological Reflection: The Evolution of Epistemological Assumptions from Age 18 to 30. En B. Hofer, B. y P. Pintrich, (Eds.) Personal Epistemology. The Psychology of 
Beliefs About Knowledge and Knowing Mahwah, New Jersey: Lawrence Erlbaum Associates, 89102.

BLOOM, B. (1971) Taxonomía de los objetivos de la educación. La clasificación de las metas educacionales. Buenos Aires: Centro Regional de Ayuda Técnica, Agencia Internacional para el Desarrollo.

BROWNLEE, J., PURDIE, N. y BOULTON-LEWIS, G. (2001) Changing Epistemological Beliefs in PreService Teacher Education Students. Teaching in Higher Education, 6(2), 247-268.

BUEHL, M. y ALEXANDER, P. (2002) Beliefs about Schooled Knowledge: Domain Specific or Domain General? Contemporary Educational Psychology, 27, 415-449.

CALDERHEAD, J. (1996) Teachers Beliefs and Knowledge. En D. Berliner, y R. Calfee (Eds.) Handbook of Educational Psychology. New York: MacMillan Library Reference USA, (709-725.

CANNING, C. (1991) What teachers say about reflection. Education Leadership, 48(6), 18-21.

CHAN, K. y ELLIOT, R. (2002) Exploratory Study of Hong-Kong Teacher Education Student's Epistemological Beliefs: Cultural Perspectives and Implications on Beliefs Research. Contemporary Educational Psychology, 27, 392-414.

CLINCHY, B. (2002) Revisiting Women's Way of Thinking. En B. Hofer y P. Pintrich (Eds.) Personal Epistemology. The Psychology of Beliefs about Knowledge and Knowing. Mahwah, New Jersey: Lawrence Erlbaum Associates, 63-87.

COLLIER, S. (1999) Characteristics of Reflective Thought During the Student Teaching Experience. Journal of Teacher Education, 50(3), 173-181.

COX, C. (1999) La reforma del curriculum. En J. García-Huidobro (Ed.) La reforma educacional chilena. Madrid: Editorial Popular, 233-266.

DANCY, J. (2001) Problemas de la Epistemología. En T. Honderich (Ed.) Enciclopedia Oxford de Filosofía. Madrid: Technos, 315-318.

DE CORTE, E. (2003) Learning and Instruction: Current Issues. Conferencia de apertura del Taller Internacional Temas actuales en Aprendizaje y Enseñanza, Santiago de Chile, Universidad Católica Cardenal Raúl Silva Henríquez, 2-4 de junio.

DELORS, J. (1996) La educación o la utopía necesaria. En J. Delors (Ed.) La Educación encierra un tesoro. Informe a la UNESCO de la Comisión Internacional sobre la educación en el siglo XXI. Compendio. París: Ediciones UNESCO, 9-32.

ERAUT, M. (1985) Knowledge creation and knowledge use in professional contexts. Studies in Higher Education, 10, 117-133.

FERRÁN, M. (1996) SPSS para Windows. Programación y análisis estadístico. Madrid: McGraw-Hill.

Freeman, D. (1991) To make the tacit explicit: Teacher education, emerging discourse, and conceptions of teaching. Teaching and Teacher Education, 7(5/6), 439-454.

GARCÍA-HUIDOBRO, J. y COX, C. (1999) La reforma educacional chilena 1990-1998. Visión de conjunto. En J. García-Huidobro, J. La reforma educacional chilena. Madrid: Editorial Popular, 7-46. 
GINSBURG, M. y NEWMAN, K. (1985) Social inequalities, schooling, and teacher education. Journal of Teacher Education, 36(2), 49-54.

HAMMER, D. y ELBY, A. (2002) On the Form of a Personal Epistemology. En B. Hofer y P. Pintrich (Eds.) Personal Epistemology. The Psychology of Beliefs about Knowledge and Knowing. Mahwah, New Jersey: Lawrence Erlbaum Associates, 169-190.

HARVEY, O. (1986) Belief systems and attitudes toward the death penalty and other punishments. Journal of Psychology, 54, 143-159.

HILL, L. (2000) What Does It Take To Change Minds? Intellectual Development of Preservice Teachers. Journal of Teacher Education, 51(1), 50-62.

HOFER, B. y PINTRICH, P. (1997) The Development of Epistemological Theories: Beliefs about Knowledge and Knowing and their Relation to Learning. Review of Educational Research, 67(1), 88-140.

HOFER, B. (2001) Personal Epistemology Research: Implications for Learning and Teaching. Journal of Educational Psychology Review, 13(4), 353-383.

HOFER, B. (2002) Personal Epistemology as a Psychological and Educational Construct: An Introduction. En B. Hofer y P. Pintrich (Eds.) Personal Epistemology. The Psychology of Beliefs about Knowledge and Knowing Mahwah, New Jersey: Lawrence Erlbaum Associates, 3-14.

JACKSON, P. (1968) Life in classrooms. New York: Holt, Rinehart \& Winston.

KING, P. y KITCHENER, K. (1994) Developing Reflective Judgment. San Francisco: Jossey-Bass Publishers.

KING, P. y KITCHENER, K. S. (2002) The Reflective Judgment Model: Twenty Years of Research on Epistemic Cognition. En B. Hofer y P. Pintrich (Eds.) Personal Epistemology. The Psychology of Beliefs about Knowledge and Knowing. Mahwah, New Jersey: Lawrence Erlbaum Associates, 3761.

KITCHENER, K. S. (1983) Cognition, Metacognition, and Epistemic Cognition. Human Development, 26, 222-232.

KITCHENER, R. (2002) Folk Epistemology: An Introduction. New Ideas in Psychology, 20, 89-105.

KUHN, D. y WEINSTOCK, M. (2002) What Is Epistemological Thinking and Why Does It Matter? En B. Hofer, B. y P. Pintrich (Eds.) Personal Epistemology. The Psychology of Beliefs about Knowledge and Knowing. Mahwah, New Jersey: Lawrence Erlbaum Associates, 121-140.

LEÓN, O. y MONTERO, I. (1997) Diseño de investigaciones. Introducción a la lógica de la investigación en Psicología y Educación. Madrid: MacGraw-Hill.

LONKA, K., JORAM, E. y BRYSON, M. (1996) Conceptions of Learning and Knowledge: Does Training Make a Difference? Contemporary Educational Psychology, 21, 240-260.

LORTIE, D. (1975) Schoolteacher: A sociological study. Chicago: University of Chicago Press.

MASON, L. (2004) Paul R. Pintrich y la investigación sobre creencias epistemológicas. Revista Electrónica de Investigación Psicopedagógica, 3, 163-170. 
MOORE, W. (2002) Understanding Learning in a Postmodern World: Reconsidering the Perry Scheme of Ethical and Intellectual Development. En B. Hofer y P. Pintrich (Eds.) Personal Epistemology. The Psychology of Beliefs about Knowledge and Knowing. Mahwah, New Jersey: Lawrence Erlbaum Associates, 17-36.

MOSHMAN, D. (1998) Cognitive development beyond childhood: Constraints on cognitive development and learning. En W. Damon (Series Ed.), D. Kuhn \& R. Siegler (Vol. Eds.) Handbook of child psychology: Vol. 2: Cognition, language, and perceptios ( $5^{\text {th }}$ ed.). New York: Wiley, 947-978.

NESPOR, J. (1987) The role of beliefs in the practice of teaching. Journal of curriculum studies, 18,197-206.

NÚÑEZ, I. (1999) Políticas hacia el magisterio. En J. E. García-Huidobro (Ed.) La reforma educacional chilena. Madrid: Editorial Popular, 177-194.

PAJARES, F. (1992) Teachers' Beliefs and Educational Research: Cleaning Up a Messy Construct. Review Of Educational Research, 62(3), 307-332.

PERRY, W. (1970) Forms of intellectual and ethical development in the college years: A Scheme. New York: Holt, Rinehart \& Winston.

PORTER, A. y FREEMAN, D. (1986) Professional orientations: An essential domain for teaching testing. Journal of Negro Education, 55, 284-292.

POSNER, G., STRIKE, K., HEWSON, P. y HERTZOG, W. (1982) Accomodation of a scientific conception: Toward a theory of conceptual change. Science Education, 66, 211-227.

PULTORAK, E. (1993) Facilitating reflective thought in novice teachers. Journal of Teacher Education, 44, 288-295.

PULTORAK, E. (1996) Following the developmental process of reflection in novice teachers: Three years of investigation. Journal of Teacher Education, 47, 283-291.

ROCKEACH, M. (1968) Beliefs, attitudes and values: $A$ theory of organization and change. San Francisco: Jossey-Bass.

RUDNEY, G. y GUILLAUME, A. (1990) Reflective teaching for students teachers. The Teacher Educator, 25(3), 13-20.

SCHOMMER, M. (1990) Effects of beliefs about the nature of knowledge on comprehension. Journal of Educational Psychology, 82, 498-504.

SCHOMMER-AIKINS, M. (2002) An Evolving Theoretical Framework for an Epistemological Belief System. En B. Hofer y P. Pintrich (Eds.) Personal Epistemology. The Psychology of Beliefs about Knowledge and Knowing. Mahwah, New Jersey: Lawrence Erlbaum Associates, 103-118.

SCHRAW, G. y OLAFSON, L. (2003) Teacher's Epistemological World Views and Educational Practices. Journal of Cognitive Education and Psychology, 3(2) 178-235.

SCHRAW, G., BENDIXEN, L. y DUNKLE, M. (2002) Development and Validation of The Epistemic Belief Inventory (EBI). En B. Hofer y P. Pintrich (Eds.) Personal Epistemology. The Psychology of Beliefs about Knowledge and Knowing. Mahwah, New Jersey: Lawrence Erlbaum Associates, 261-275.

SCHUTZ, A. (1970) On phenomenology and social relations. Chicago: University of Chicago Press. 
SIGEL, I. (1985) A conceptual analysis of beliefs. En I. E. Sigel (Ed.) Parental belief systems: The psychological consequences for children. Hillsdale, NJ: Erlbaum, 345-371.

SINATRA, G. (2002) Motivational, Social, and Contextual Aspects of Conceptual Change: A Commentary. En Limón, M. y Mason, L. (Eds.) Reconsidering Conceptual Change. Issues in Theory and Practice. Netherlands: Kluwer Academic Publishers, 187-197.

VEENMAN, S. (1984) Perceived problems of beginning teachers. Review of Educational Research, 54, 143178.

WILDMAN, T. y NILES, J. (1987) Reflective teachers: Tensions between abstractions and realities. Journal of Teacher Education, 38(4), 25-31.

ZHANG, L. (1999) A Comparison of U.S. and Chinese University Students' Cognitive Development: The Cross-Cultural Applicability of Perry's Theory. The Journal of Psychology, 133 (4), 425-439. 


\title{
Contactar
}

Revista lberoamericana de Educación

\author{
Principal OEI
}

